\title{
Simulation Study and Implementation of Different Mobile Ad-Hoc Network (MANET) Routing Protocol under Network Traffic
}

\author{
Neelam \\ NIET, Greater Noida
}

\author{
Pankaj Kumar \\ NIET, Greater Noida
}

\author{
Atul Gupta \\ NIET, Greater Noida
}

\begin{abstract}
The performance of different routing protocols has been widely studied. Many routing protocols for Ad-hoc networks have been proposed till now. Amongst the most popular ones are Ad-hoc on demand Distance Vector Routing Protocol (AODV), Dynamic Source Routing Protocols (DSR), and Destination Sequenced Distance Vector (DSDV).

We study the performance of routing protocols when particular application exists in the network. A source node need to send large data files to the number of nodes, using File Transfer Protocol (FTP), while other non-specific application traffic also exists in the network.

In this paper, we compare the performance of three routing protocols AODV, DSR (reactive), DSDV (proactive) under FTP traffic by varying number of relay nodes in terms of packet delivery ratio, end to end delay, normalized routing overhead. A Network Simulator (NS2) Tool is used to developed the scenario and evaluate them on the basis of performance metrics.
\end{abstract}

\section{Keywords}

Routing Protocols, DSDV, DSR, AODV, FTP, NS2

\section{INTRODUCTION}

An ad hoc network is a collection of wireless mobile nodes (or routers) dynamically forming a temporary network without the use of any existing network infrastructure or centralized administration $[15,16]$. Nodes are free to move randomly and organize themselves arbitrarily having a dynamically changing topography. Each node acts as a transmitter, receiver or router. Mobile Ad-hoc Networks (MANETs) [1] use many different routing protocols to route data packets between nodes.

Most proposing routing protocols for MANET can be classified into three different categories: proactive (table driven) routing protocol, reactive (on demand) routing protocol and hybrid routing protocol [2]. In proactive routing protocols, each node maintains one or more routing table containing the routing information to every other node in a network. All the nodes update routing tables in order to maintain the consistent and up-to date view of the network. Proactive routing protocols are also called the table driven routing protocol. Some of the popular protocols examined in previous studies Optimized Link State Routing (OLSR), Destination-Sequenced Distance Vector (DSDV) and Wireless Routing Protocol (WRP), which belong to the table driven category $[15,16]$.

Reactive routing protocols are slow approach to routing than the proactive routing protocols.
In Reactive protocols, nodes try to build a route only when a source wants to send packets to a destination following the route discovery mechanisms. The route will remain valid as long as the destination is reachable. Reactive routing protocols are also called the On Demand routing protocols. Some of the most popular protocols examined in previous studies are Dynamic Source

Routing (DSR), Ad-hoc On -Demand Distance Vector (AODV) and Temporally-Ordered Routing Algorithm (TORA), these belong to on -demand category. There are series of studies to compare the performance evaluation that examine the performance and operation of these protocols and comparing them in terms of various metrics [3-13].

In this study we examine the operation and performance of the most popular routing protocols in a case study, where a common source node (server) need to send large data files to varying number of nodes. Later work has been done in this area uses traffic generators that do not correspond to a particular application .when FTP traffic exists in the network ,the performance of the routing protocols could be prominently different than in simple scenario studied in the past, which do not apply in real situations. The routing protocols under our investigation are DSR $[3,4,6]$, AODV [3, $5,7]$, DSDV [3, 6, 9]. DSR and AODV are reactive routing protocol while DSDV is a proactive routing protocol. They all are used in flat network topologies.

The rest of the paper is organized as follows. Section 2 describes an overview of related work. In Section 3 we describe the case study scenario. In section 4 , the simulation results are presented. In section 5 , the conclusion and future scope are discussed.

\section{RELATED WORK}

A number of routing protocols have been implemented and proposed for mobile ad-hoc network in order to enhance the more throughput, the bandwidth usage, less overheads per packet, least consumption of energy and others. In this section, the traffic sources were considered to send Constant Bit Rate (CBR) traffic and not resulting from a specific application. In this part we present some of the useful work done in this field in contrast to our study.

In [3], AODV, DSR and DSDV are compared by varying network load, mobility model and measuring the performance metrics such as packet delivery ratio, average end to end delay, and packet dropped.

In [4], evaluate the performance of DSR, OLSR and ZRP protocols in terms of end- to-end delay, average jitter, average throughput, normalized routing load and packet delivery fraction. 
The mobility model used is random waypoint model. This paper focuses on to analyze the performance of popular MANETs routing protocol in high mobility scenarios.

In [5], OLSR, AODV, GRP are compared using three parameters such that throughput, delay and network load under FTP traffic and HTTP traffic.

In [6], evaluate the performance of AODV, DSR, DSDV protocols with multimedia traffic source in the network based on the following performance metrics: energy consumption, jitter, packet delivery ratio, packet drop, control overhead and delay. The network simulator 2 (NS -2.35) tool is used to study the scenario and evaluate them.

In [7], AODV and TORA are compared in terms of traffic delay and traffic deliver rate. This paper focuses on choosing routing protocols for network with a composite traffic model that is closer to the real situation.

In [9], evaluate and compare the performance of four routing protocols DSDV, OLSR, DSR and AODV for CBR, FTP traffic by varying number of nodes in terms of throughput, end to end delay and packet loss.

In [11], DSR, AODV and WRP are compared in terms of different performance metrics which are average end to end delay, packet delivery ratio, routing message overhead and throughput by varying pause time, offered load and average node speed. Other types of traffic, besides CBR are examined, namely FTP and TELNET traffic.

In [13], DSR, AODV and OLSR are compared in terms of packet delivery ratio, average end to end delay and routing overhead when FTP traffic coexists in the network, with other non-specific application traffic.

We examine that DSR, AODV and DSDV are compared by varying the number of the relay nodes and the number of nodes requesting files from the source. We study the performance in terms of average end to end delay, normalized routing overhead and packet delivery ratio of above mentioned routing protocols.

\section{CASE STUDY}

In this section we describe the case study scenario which is used for setup and execution of the simulation. The dimensions of simulation field are $1100 \mathrm{~m} \times 1100 \mathrm{~m}$. There are a static source node placed on the left edge of the field and static destination nodes randomly placed on the right edge of the field.

Here we execute two sets of simulations. In the first one, FTP traffic coexists with non-specific application traffic and in the second one, there is non-specific application traffic in the network using CBR and burst traffic generators. In both of simulation is used the whole traffic generators' configuration is adapted to maintain the total load of the network is same. In first one, large files are transferred from the source node to each of destination nodes using FTP during whole simulation. In the field there is a number of randomly moving nodes that are going to be used as relay nodes between the source and destination nodes. The transmission range of each node is set to $200 \mathrm{~m}$. All of the main simulation parameters are defined in the table 1 .

Table 1: Simulation Parameters

\begin{tabular}{|l|l|}
\hline \multicolumn{1}{|c|}{ Parameter } & \multicolumn{1}{c|}{ Value } \\
\hline Simulation area & $1100 \mathrm{~m} \times 1100 \mathrm{~m}$ \\
\hline Simulation Time & $150 \mathrm{~s}$ \\
\hline
\end{tabular}

\begin{tabular}{|l|l|}
\hline Radio Propagation Model & Two-Ray Ground model \\
\hline Transmission Range & $200 \mathrm{~m}$ \\
\hline Routing Protocols & DSR , AODV , DSDV \\
\hline Packet Size & 512 bytes \\
\hline Application & FTP \\
\hline $\begin{array}{l}\text { Number of FTP source } \\
\text { nodes }\end{array}$ & 1 \\
\hline $\begin{array}{l}\text { Number of FTP destination } \\
\text { nodes }\end{array}$ & $1,3,5,7$ \\
\hline Number of relay nodes & $5,10,15,20$ \\
\hline Mobility Models & Random Way Point \\
\hline MAC & 802.11 \\
\hline Relay Nodes, Speed & $2-10 \mathrm{~m} / \mathrm{s}$ \\
\hline
\end{tabular}

We generated different network scenario for increasing number of nodes and performance metrics considered for evaluation are:

Packet Delivery Ratio (PDR): PDR is the fraction of total packet sent to the total packets received.

Average End-to-End Delay (AEED): AEED is the average time a data packet needs to be delivered to its destination.

Normalized Routing Overhead (NRO): It is ratio between control traffic and total throughput of the network.

These are the mostly used and they are selected for easier comparison to which work already done on this field. For all the above-mentioned metrics the total amount of traffic is considered for the calculations.

\section{SIMULATION RESULTS}

In Fig. 1, the PDR versus the number of relay nodes is depicted for DSR, AODV and DSDV for different number of FTP destination nodes. In Fig. 2, the average End-to-End Delay can be observed. Finally, the Normalized Routing Overhead is presented in the same way in Fig. 3.

Dashed lines represent the value of respective metric when only non-specific application traffic exists; compact lines represent the same metric when FTP traffic coexists with nonspecific application traffic exists in the network. In both simulation and set up and total network load has been computed in the simulation to be same. All metrics are depicted versus the number of relay node for easier comparison.

As we expected the performance of the network degrades when more traffic is present in the network destined towards more FTP destination nodes. Additionally, when the network becomes of high density there is significant impact on all of the three protocols' performance metrics that we considered in this study. As the traffic load and density of network increases, the PDR decreases, while the average End-to-End Delay increases, for all the protocols under consideration. This happens because of the increased probability of collisions and packet drops due to network congestion, when traffic load and network density increases. Normalized routing overhead also increases as the number of relay nodes and the amount of traffic increases, but not regularly.

We evaluated the performance of AODV, DSR and DSDV protocols for different number of relay nodes 5, 10, 15 and 20 for different number of FTP destination nodes with FTP traffic. The performance metrics to be compared are average end- to- end delay, Packet Delivery Ratio and normalized routing overhead. 


\subsection{Packet Delivery Ratio}

Figure 1(a, b, c, d) shows packet delivery ratio versus number of relay nodes for AODV, DSR and DSDV for different FTP destination node. Dashed lines represent the value of respective metric when only non-specific application traffic exists; compact lines represent the same metric when FTP traffic coexists with non-specific application traffic exists in the network. It is conclude that packet delivery ratio is more in AODV as compared to DSR and DSDV. AODV is best and DSDV is worst among all the protocols in packet delivery ratio which makes AODV more preferable.

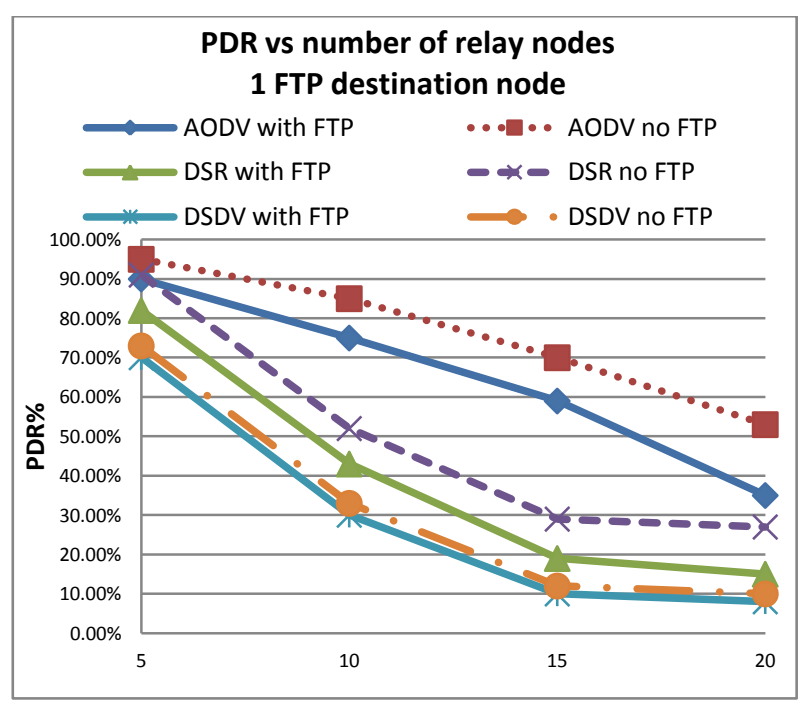

Figure 1 (a): PDR vs. Number of Relay Nodes for 1FTP Destination node

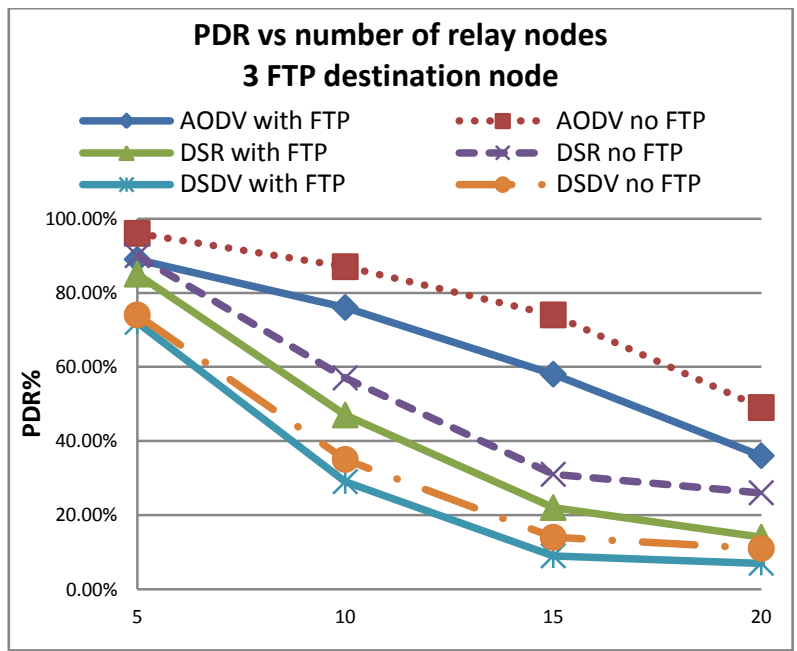

Figure 2 (b): PDR vs. Number of Relay Nodes for 3 FTP Destination nodes

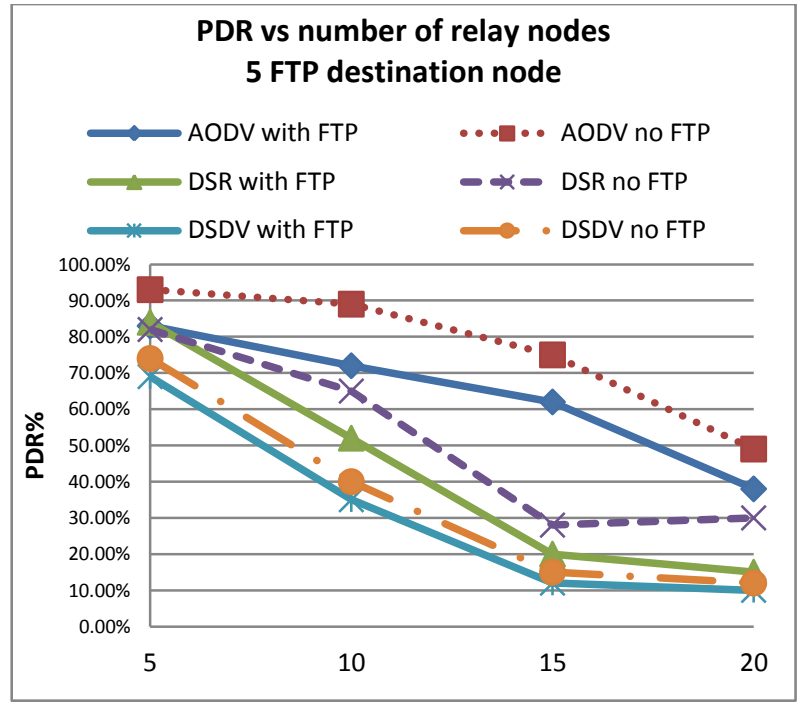

Figure 3 (c): PDR vs. Number of Relay Nodes for 5 FTP Destination nodes

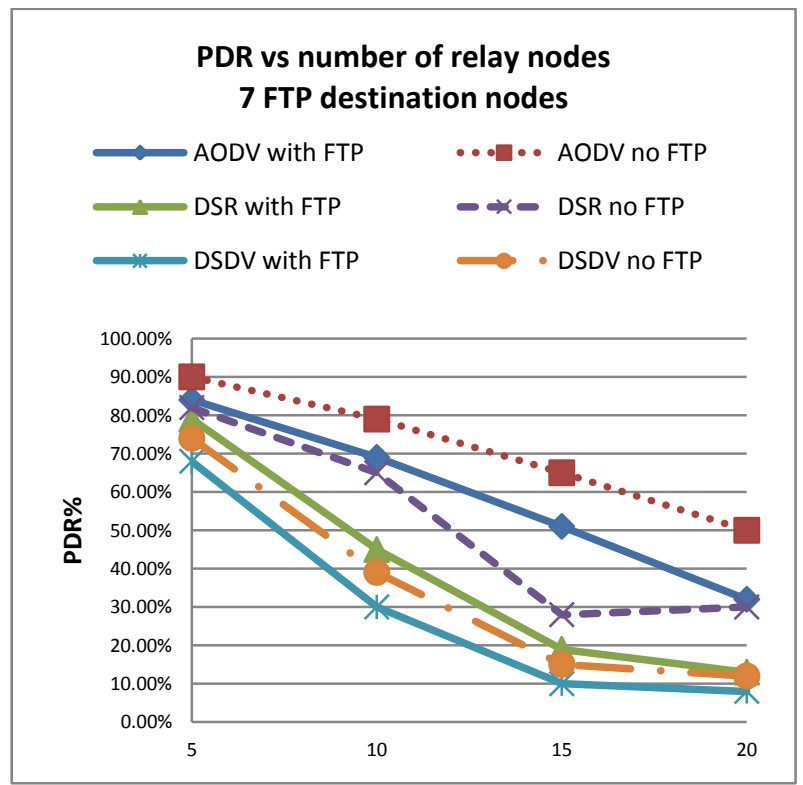

Figure 4 (d): PDR vs. Number of Relay Nodes for 7 FTP Destination nodes

\subsection{Average End -To- End Delay}

Figure 2 (a, b, c, d) shows average end -to-end delay versus number of relay nodes for AODV, DSR and DSDV for different FTP destination node. Dashed lines represent the value of respective metric only non-specific application traffic exists, compact lines represent the same metric when FTP traffic coexists with non-specific application traffic exists in the network. It concludes that average end to end delay is very less and remains almost same for all the nodes in case of DSDV. The performance of AODV has less end to end delay compared to DSR. 


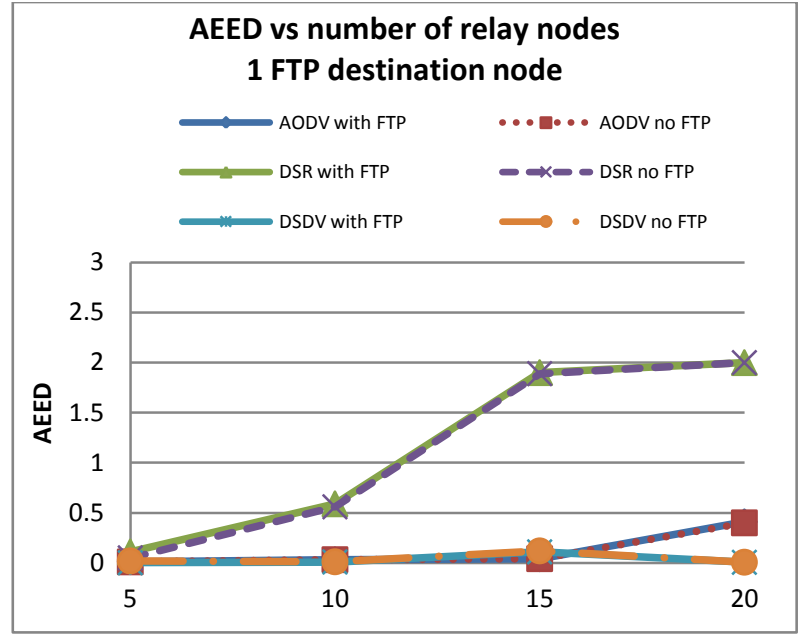

Figure 2 (a): AEED vs. Number of Relay Nodes for 1 FTP Destination node

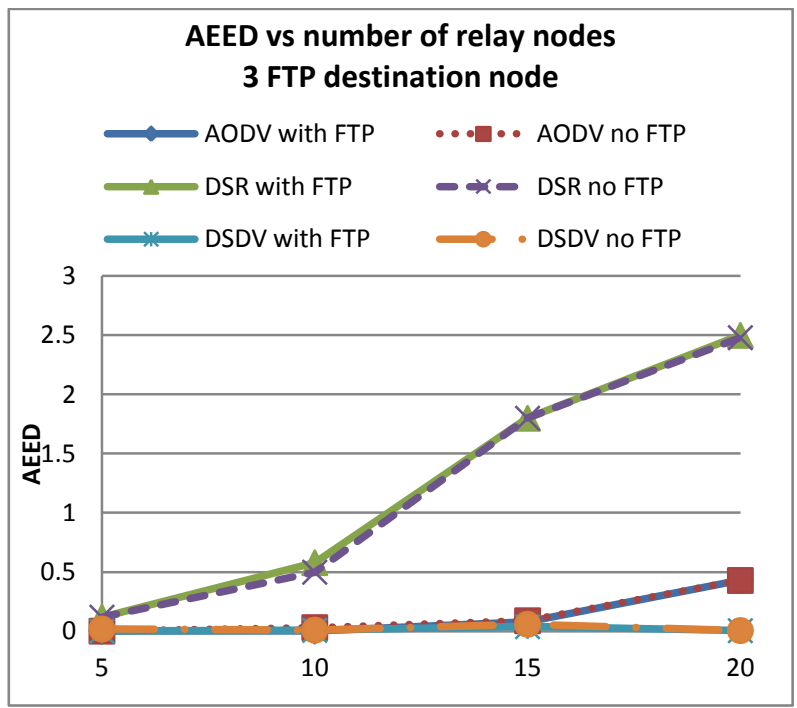

Figure 2 (b): AEED vs. Number of Relay Nodes for 3 FTP Destination nodes

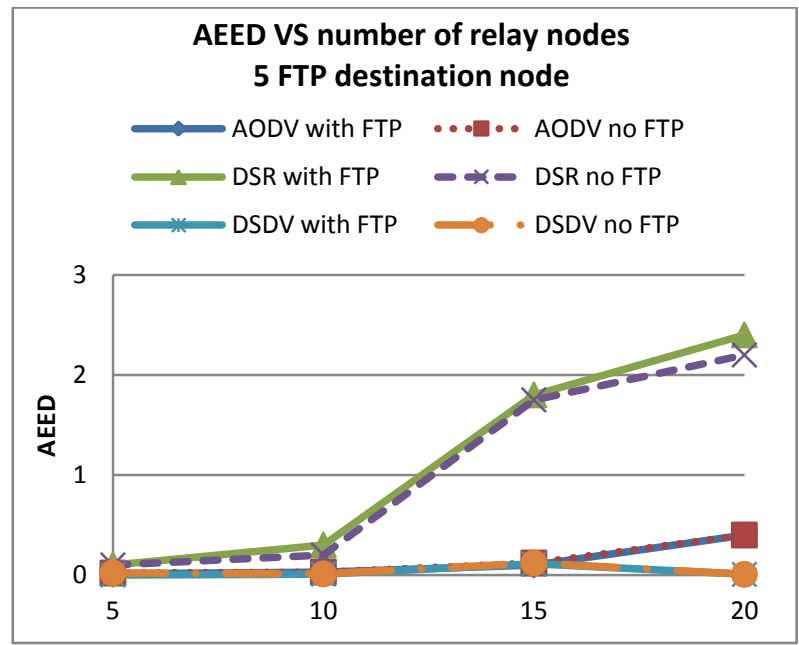

Figure 2 (c): AEED vs. Number of Relay Nodes for 5 FTP Destination nodes

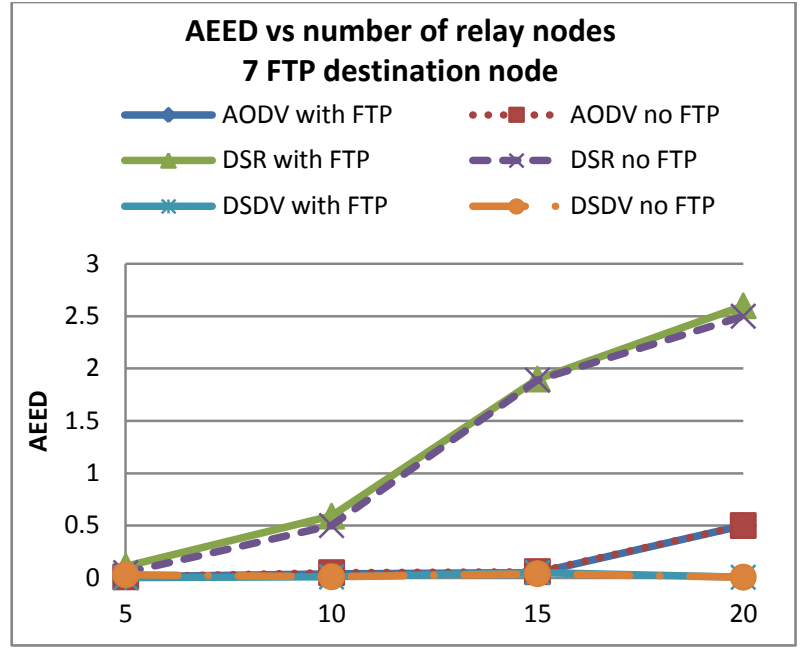

Figure 2 (d): AEED vs. Number of Relay Nodes for 7 FTP Destination nodes

\subsection{Normalized Routing Overhead}

Figure 3(a, b, c, d) shows normalized routing overhead versus number of relay nodes for AODV, DSR and DSDV for different FTP destination node. A set of simulations was performed without the presence of FTP traffic but with nonspecific application traffic in order to maintain the total load of the network equivalent to the load with FTP source and destination nodes. On the basis of normalized routing overhead, DSDV seems to be better than all the routing protocols. The NRO of DSDV is not affected by the density of the relay nodes in the network slightly affected by number of FTP flows that are present in the network.

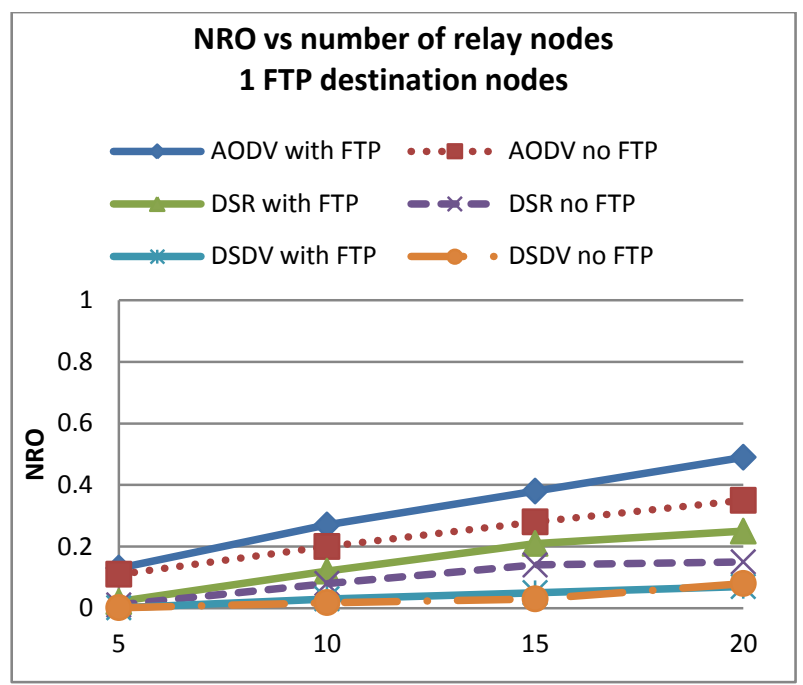

Figure 3 (a): NRO vs. Number of Relay Nodes for 1 FTP Destination node 


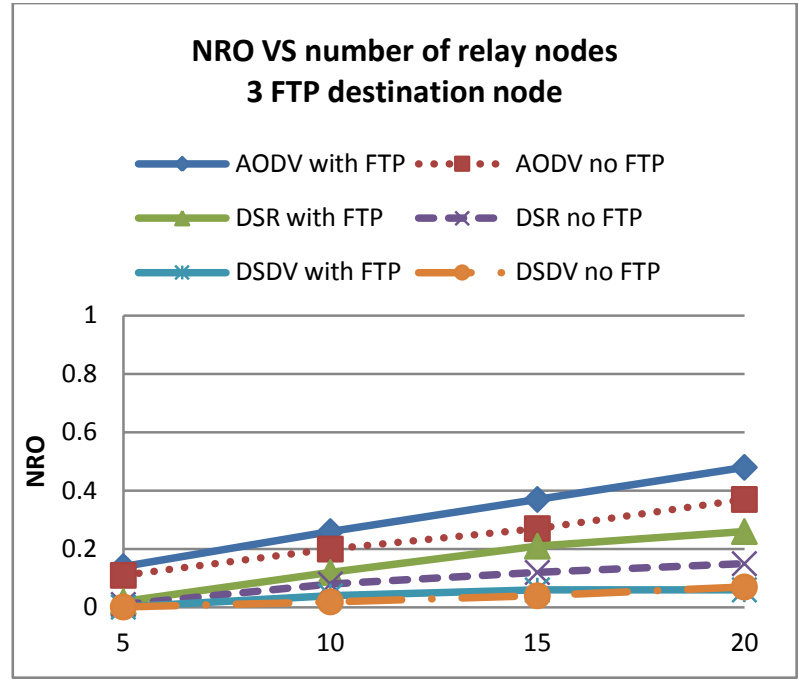

Figure 3 (b): NRO vs. Number of Relay Nodes for 3 FTP Destination nodes

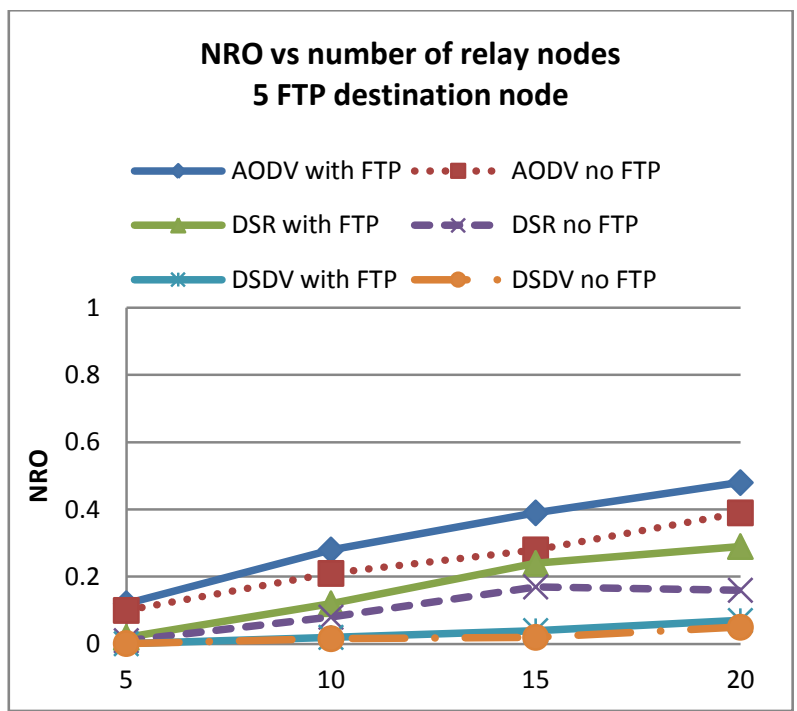

Figure 3 (c): NRO vs. Number of Relay Nodes for 5 FTP Destination nodes

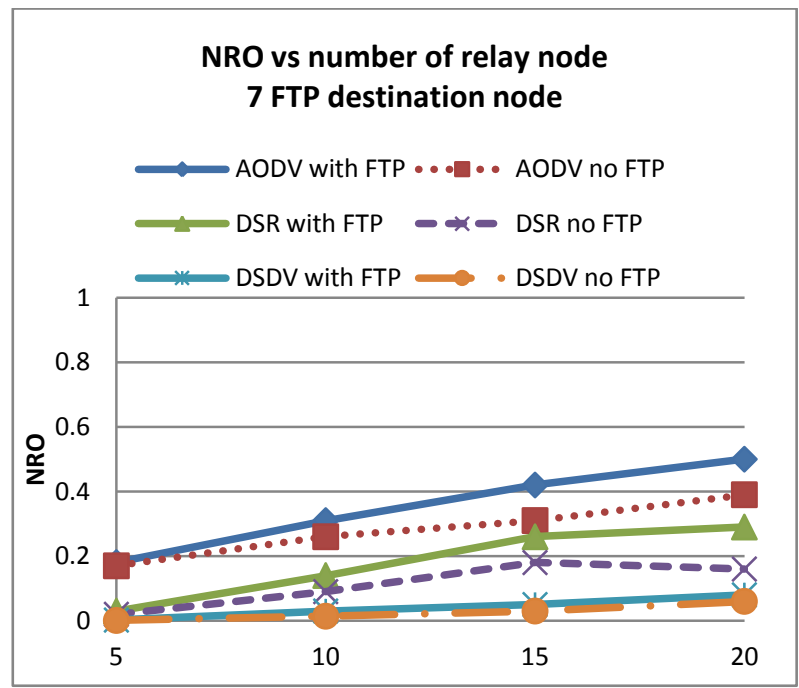

Figure 3 (d): NRO vs. Number of Relay Nodes for 7 FTP Destination nodes

\section{CONCLUSION AND FUTURE SCOPE}

In this paper, we compare the performance of three routing protocol AODV, DSR, DSDV using network simulator (NS2.35). We compared the result of a simulation where FTP traffic was absent and when FTP traffic exists in the network but total traffic load was equal in whole simulation. We conclude that the routing protocols have better performance in terms of PDR and NRO for all cases but AEED remains same in the same level as when ftp traffic coexists in the network. In FTP traffic the value of PDR is small as for DSR and DSDV for all network size as compared to AODV which makes AODV more favorable.

In terms of NRO, DSR and DSDV are best as compared to the AODV. In FTP traffic the value of average delay is small for AODV and DSDV as compared to DSR which makes AODV and DSDV more favorable. AODV performance is adequate in all network densities and traffic loads, while overhead is maintained at reasonable levels. Finally, the type of traffic has a significant impact on the performance of three protocols under investigation. In future we expand our study towards hybrid routing protocols, plan to investigate the impact of different multimedia traffic, using more performances metrics and more difficult scenario.

\section{REFERENCES}

[1] Subir Kumar Sarkar, T. G. Basavaraju and C. Puttamadappa "Ad hoc mobile wireless networks: principles, protocols, and applications," Auerbach Publications 2008.

[2] Neelesh Gupta and Roopam Gupta "Routing Protocols in Mobile Ad-Hoc Networks: an Overview" 2010 IEEE.

[3] Laxmi Shrivastava, Sarita S. Bhadauria, G. S. Tomar "Performance Evaluation of Routing Protocols in MANET with different traffic loads" 2011 International Conference on Communication Systems and Network Technologies, IEEE.

[4] Savita Gandhi, Nirbhay Chaubey, Pathik Shah and Madhvi Sadhwani "Performance Evaluation of DSR, OLSR and ZRP Protocols in MANETs" ICCCI -2012, Jan. 10 - 12, IEEE.

[5] Nirendra Kumar, Pratap Singh Patwal "Performance Evaluation of MANET Routing Protocols of with FTP Application Using an Optimized Scalable Simulation Model,"2013, IJARCSSE.

[6] Ajinkya. D. Kadam, Sharad .S .Wagh "Evaluating MANET Routing Protocols under Multimedia Traffic," July 4-6, 2013, IEEE.

[7] An Ran, Qu Jinxin and ShaXuejun "MANET Routing Protocols Comparison for Composite Traffic Network" 2013 Third International Conference on Instrumentation, Measurement, Computer, Communication and Control, IEEE.

[8] Greis, M. Tutorial for the Network Simulator "NS" 2004

[9] Katroth Balakrishna Maruthiram and Kare Suresh Babu "Performance Comparison of DSDV, OLSR, DSR and AODV MANET Routing Protocols in Traffic Conditions"IJSR, Volume 3 Issue 8, August 2014.

[10] M.L Sharma, Noor Fatima Rizvi, Nipun Sharma, Anu Malhan and Swati Sharma "Performance Evaluation of MANET Routing Protocols under CBR and FTP traffic classes". 
[11] Arora, V.; Rama Krishna, C., Performance evaluation of routing protocols for MANETs under different traffic conditions," Computer Engineering and Technology (ICCET), 2010 2nd International Conference on , vol.6, no., pp.V6-79,V6-84, 16-18 April 2010.

[12] The ns Manual (formerly ns Notes and Documentation). UC Berkeley, LBL, USC/ISI, and Xerox PARC; 2011.

[13] Dimitra Kampitaki and Anastasios A. Economides, "Simulation study of MANET routing protocols under FTP traffic," Conference on Electronics, Telecommunications and Computers (CTEC), ELSEVIER COMPUTER NETWORKS, 2014.
[14] Anuj K. Gupta ,Harsh Sadawarti and Anil K.Verma, "Review of Various Routing Protocols for MANETs" International Journal of Information and Electronics Engineering, Vol. 1, No. 3, November 2011.

[15] Saleh Ali K. Al-Omari, Putra Sumari, “An Overview Of Mobile Ad-hoc Networks for the Existing Routing Protocol and Applications"vol.2, No.1, march 2010.

[16] Aaliya Manzoor, Virender Sharma "A Survey of Routing and Mobility Models for Wireless Ad hoc Network" SSRG-IJCSE -EFES in April 2015. 